\title{
Evaluation of Hydroxychloroquine Effect on the Retinal Layers by Spectral-Domain Optical Coherence Tomography
}

\author{
A.H.El Habbak, O.M.Kamal, A.A.Shebl and M.E.El Tahlawy
}

Ophthalmology, Dept., Faculty of Medicine, Benha Univ., Benha, Egypt

E-mail:mahmoudtaa7777@gmail.com

\begin{abstract}
Objective: Despite reduced systemic toxicity compared to other medicines, hydroxychloroquine is a widely used immunosuppressive agent for the treatment of different autoimmune disorders. It may induce serious retinal impairment and blindness. The purpose of this research is to evaluate the early OCT results in preclinical retinopathy of hydroxychloroquine (HCQ). Patients and procedures: This cross-sectional comparative study has taken place in ophthalmology and rheumatology clinics in Benha university hospitals; twenty eyes of rheumatic HCQ patients for over two years and 20 eyes of ordinary gender patients have been examined and imaged fully with spectrum optic coherence tomography (SD-OCT). The whole research group had a normal look of a fundus. Results: For more than two years of usage with still normal fundus, external retinal dilation as central foveal and parafoveal dilution was observed with SD-OCT in individuals with chronic HCQ. In just 2 rheumatic individuals the IS/OS junction was disrupted (10 percent ). Only three rheumatic patients suffered from colour vision (15 percent ). Conclusion: Preclinical HCQ retinopathy may be detected in the screening of SD-OCT rheumatic patients, particularly with the increasing availability of SD-OCT machinery in most visual centres.
\end{abstract}

Key words: Retinopathy, hydroxychloroquine, Spectral domain OCT.

\section{Introduction}

Hydroxychloroquine is a widely used immunosuppressives agent, despite less systemic toxicity than other medications, for the treatment of different autoimmune disorders and may induce serious retinal degeneration and vision loss. Retinal toxicity can occur as a secondary effect of long-term hydroxychloroquine therapy, high dose and long-term use are the most important risk factors, dose $>5.0$ $\mathrm{mg} / \mathrm{kg}$ dramatically increases both the population risk and annual incremental risk, and extreme doses can be extremely dangerous, other main factors being concurrent renal illness or tamoxifen use [1].

The main pathogens in Hydroxychloroquine are lysosomal dysfunctions in photoreceptors and RPE cells that cause RPE to build up lipofuscin, enhance melanin binding and contribute to or prolong the toxic effects of the medication in the retina. RPE is the main pathogen. The macular location shows that light absorption or cone metabolism may have a role [2].

The early identification of the medicine may avoid irreversible harm, therefore it is essential to evaluate patients for the early detection of asymptomatic retinal structural changes [3].

Recommendations for the testing of retinopathy of chloroquine and hydroxychloroquine were made public by the American Academy of Ophthalmology in 2016; the primary tests of the testing include automated visual fields plus optical coherence tomography (SD-OCT), the multifocal electroretinogram (MFERG) can provide objective corroboration for the visual field; the fundus autofluorescence (FAF) can show damages.

Modern screening should identify retinopathy before it is evident in the fundus, SD-OCT is the most utilised retinal structural change detection instrument in such examinations. Therefore, the research examines the early indications of retinal toxicity, the early abnormalities of the inner (IS), outer segment (SO) and the complex thicknesses of the membrane of RPE-Bruch using SD-OCT images[1].

SD-OCT is a highly sensitive and reproductive imaging model commonly used in clinical practical practise that is capable of detecting typical macular changes in HCQ toxicity, including loss of the parafoveal ellipsoid area (EZ), parafoveal thinning of the outer atomic (ONL), and inner plexiform layer (IPL), flying saucer sign and fibromyal peripapillary nerve (peripapillary fibre)

In HCQ retinopathy, loss of photoreceptor innerouter segment junction (the IS/OS line, which is also called ellipsoid zone or photoreceptor integrity line, PIL) and thinning of the external nuclear retina layer are noticed.[5] SD-OCT tests visualises the retinal layers in as much detail as the microscopic examination.

In this research, hydroxychloroquine was evaluated on macular retinal layers by utilising a tomography of the optical coherence of the spectral domain (SD-OCT)

\section{Patients and procedures}

This future cohort research was conducted from March 2019 to April 2021 at Banha University Hospitals.

The protocol was accepted by the scientific Committee of the Department of Ophthalmology and the Ethical Committee: Faculty of Medicine, University of Banha. All subjects received informed consents. The researcher and supervisors are not interested financially in the commercial goods described in the thesis.

The research was conducted using 40 eyes from 40 participants selected from the Banha University Hospitals Ophthalmology and Rheumatology Clinics. 


\subsection{Subjects were divided into two groups}

- Hydroxyhloroquine group: 20 eyes of 20 patients treated with hydroxychloroquine for more than 2 years with normal fundus examination.

- Control group: 20 eyes of 20 normal subjects with completely normal ophthalmological examination of age and sex matched participants.

\subsection{Inclusion criteria}

- Patients on Hydroxychloroquine for rheumatic diseases for more than 2 years.

- Good candidates for OCT imaging (no media opacity)

- No posterior segment pathology

- Non significant refractive error.

- No chronic uveitis.

- No history of glaucoma, retinopathy, retinal therapy, or other intraocular surgical intervention

- Not on other medication affecting the Retina.

2.3. Exclusion criteria

- People who refuse to give consent.

- Any posterior segment pathology.

- Significant refractive error.

- chronic uveitis.

- History of glaucoma, retinopathy, retinal therapy, or other intraocular surgical intervention

2.4. All patients underwent examination through: History taking, and complete ophthalmologic examination was done, including:

- Pupillary reaction.

- Best corrected visual acuity (BCVA) using snellen's chart testing.

- Anterior segment assessment by slit lamp examination.

- Intraocular pressure measurement with an applanation tonometer.

- Fundus examination using +20 D lens (to evaluate the periphery of the retina) and $+90 \mathrm{D}$ lens (biomicroscopy for evaluating the posterior pole).

- Spectral-Domain Optical Coherence Tomography (Structural Assessment): Pupils dilated for OCT examination in all subjects with $1 \%$ cyclopentolate. OCT was done using 3D OCT $2 \mathrm{OOO}$ FA was used (TOPCON CORPORATION, Tokyo, Japan), 3D macula mode and 5 lines cross scan mode will be used to obtain high quality images.

\subsection{Statistical Analysis}

Data were collected, coded, revised and entered to the Statistical Package for Social Science (IBM SPSS) version 20. The data were presented as number and percentages for the qualitative data, mean, standard deviations and ranges for the quantitative data with parametric distribution and median with inter quartile range (IQR) for the quantitative data with non-parametric distribution. Chi-square test was used in the comparison between two groups with qualitative data and Fisher exact test was used instead of the Chi-square test when the expected count in any cell found less than 5. Independent t-test was used in the comparison between two groups with quantitative data and parametric distribution and Mann-Whitney test was used in the comparison between two groups with quantitative data and non-parametric distribution. The confidence interval was set to $95 \%$ and the margin of error accepted was set to $5 \%$. So, the pvalue was considered significant as the following: $\mathrm{P}>$ 0.05: Non significant (NS), P < 0.05: Significant (S), $\mathrm{P}<0.01$ : Highly significant (HS).

\section{Results}

In this study, 19 patients (95\%) were females and only 1 patient was male, mean of age was 43.55 among HCQ group, So there was no statistically significant difference between studied groups among demographic data, table (1)

Table (2) shows that slit lamp exam of all patients was Free, color vision of 3 patients $(15 \%)$ was desaturated, of 17 patients (85\%) was normal among HCQ group, IS $\backslash O S$ of 2 patients (10\%) was interrupted, of 18 patients $(90 \%)$ was Intact among HCQ group, so there was no statistically significant deference between slit lamp exam, Color vision and IS/OS among control group in comparison to HCQ group.

Table (1) Demographic data among studied groups.

\begin{tabular}{|c|c|c|c|c|c|c|c|}
\hline & \multicolumn{2}{|c|}{$\begin{array}{c}\text { HCQ group } \\
(\text { No.=20) }\end{array}$} & \multicolumn{2}{|c|}{$\begin{array}{l}\text { Control group } \\
(\text { No. }=\mathbf{2 0})\end{array}$} & \multicolumn{2}{|c|}{$\begin{array}{l}\text { Chi square test/ } \\
\text { Independent t test }\end{array}$} \\
\hline & & No & $\%$ & No & $\%$ & $\mathbf{x}^{2} / \mathbf{t}^{*}$ & p value \\
\hline \multirow{2}{*}{ Sex } & Female & 19 & $95.0 \%$ & 19 & $95.0 \%$ & \multirow{2}{*}{0.000} & \multirow{2}{*}{1.000} \\
\hline & Male & 1 & $5.0 \%$ & 1 & $5.0 \%$ & & \\
\hline Age & Mean \pm SD & 43.55 & 15.42 & 41.30 & 13.43 & $0.492 *$ & 0.626 \\
\hline
\end{tabular}

Table (2) slit lamp examination, Color vision and IS/OS among studied groups.

\begin{tabular}{|c|c|c|c|c|c|c|c|}
\hline & & \multirow{2}{*}{$\begin{array}{c}\text { HCQ group } \\
\text { No }\end{array}$} & \multirow{2}{*}{$\begin{array}{c}(\text { No. }=20) \\
\%\end{array}$} & \multicolumn{2}{|c|}{ Control group $($ No. $=20)$} & \multicolumn{2}{|c|}{ Chi square test } \\
\hline & & & & No & $\%$ & $\mathbf{x}^{2}$ & p value \\
\hline SLE (slit lamp exam) & NAD & 20 & $100.0 \%$ & 20 & $100.0 \%$ & NA & NA \\
\hline Color vision & $\begin{array}{c}\text { Desaturation } \\
\text { Good }\end{array}$ & $\begin{array}{c}3 \\
17\end{array}$ & $\begin{array}{l}15.0 \% \\
85.0 \%\end{array}$ & $\begin{array}{c}0 \\
20\end{array}$ & $\begin{array}{c}0.0 \% \\
100.0 \%\end{array}$ & 3.243 & 0.198 \\
\hline IS/OS & $\begin{array}{c}\text { interrupted } \\
\text { Intact }\end{array}$ & $\begin{array}{c}2 \\
18\end{array}$ & $\begin{array}{l}10.0 \% \\
90.0 \%\end{array}$ & $\begin{array}{c}0 \\
20\end{array}$ & $\begin{array}{c}0.0 \% \\
100.0 \%\end{array}$ & 2.105 & 0.146 \\
\hline
\end{tabular}


Fig. (1) shows that mean of central foveal thickness was $199.50 \mu \mathrm{m}$ among HCQ group. So there was statistically significant decrease CFT (central foveal thickness) in HCQ group in comparison to control group.

Table (3) shows that mean of Superior parafoveal was $289.75 \mu \mathrm{m}$, mean of Inferior parafoveal was $280.35 \mu \mathrm{m}$, of Nasal parafoveal was $276.25 \mu \mathrm{m}$, of Temporal parafoveal was $269.20 \mu \mathrm{m}$ among HCQ group. So there was statistically significant decrease Superior parafoveal, Inferior parafoveal, Nasal parafoveal and Temporal parafoveal in HCQ group in comparison to control group.
Table (4) shows that mean of Superior perifoveal was $257.50 \mu \mathrm{m}$, mean of Inferior perifoveal was $260.95 \mu \mathrm{m}$, of Nasal perifoveal was $269.90 \mu \mathrm{m}$, of Temporal perifoveal was $245.35 \mu \mathrm{m}$ among HCQ group.So there was no statistically significant difference between HCQ group and control group among Perifoveal region thickness.

Table (6) shows that Cumulative dose (grams) has negative correlation with parafoveal quadrants thickness $(\mathrm{r}=0.001)$ in HCQ group

Table (7) shows that treatment duration has negative correlation with CFT $(r=0.003)$, superior parafoveal $(\mathrm{r}=0.020)$ and temporal parafoveal $(\mathrm{r}=$ 0.013 ) in HCQ group

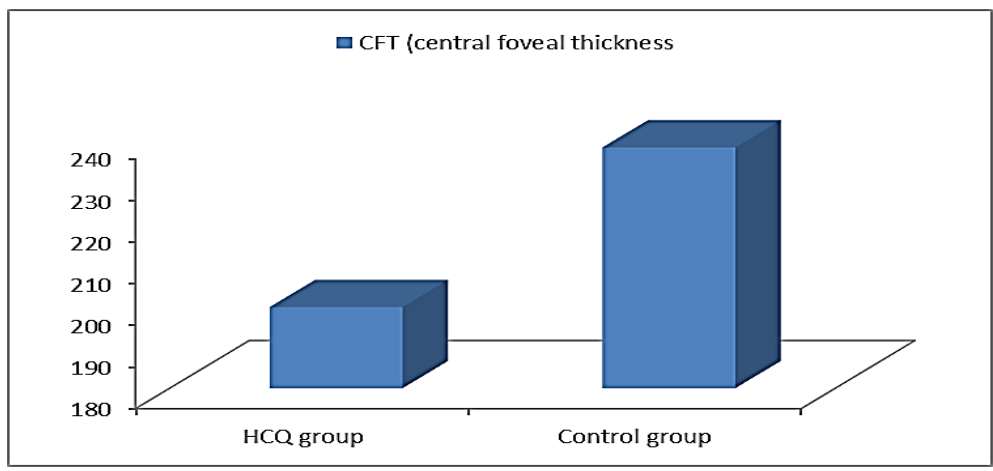

Fig. (1) CFT among studied groups.

Table (3) Parafoveal thickness among studied groups.

\begin{tabular}{lcccccc}
\hline & \multicolumn{2}{c}{$\begin{array}{c}\text { HCQ group } \\
(\text { No.=20) }\end{array}$} & \multicolumn{2}{c}{$\begin{array}{c}\text { Control group } \\
\text { (No.=20) }\end{array}$} & \multicolumn{2}{c}{ Independent t test } \\
& Mean & SD & Mean & SD & T & p value \\
\hline Superior parafoveal & 289.75 & 15.81 & 301.75 & 12.06 & $2.699-$ & 0.010 \\
Inferior parafoveal & 280.35 & 18.59 & 302.20 & 25.38 & $3.106-$ & 0.004 \\
Nasal parafoveal & 276.25 & 30.44 & 302.00 & 10.73 & $3.569-$ & 0.001 \\
Temporal parafoveal & 269.20 & 20.60 & 288.00 & 10.43 & $3.641-$ & 0.001 \\
\hline
\end{tabular}

Table (4) Perifoveal thickness among studied groups.

\begin{tabular}{lllllll}
\hline & $\begin{array}{l}\text { HCQ group } \\
\text { (No.=20) }\end{array}$ & \multicolumn{2}{l}{$\begin{array}{l}\text { Control group } \\
\text { (No.=20) }\end{array}$} & \multicolumn{2}{l}{ Independent t test } \\
& Mean & SD & Mean & SD & T & p value \\
\hline Superior perifoveal & 257.50 & 11.01 & 259.15 & 10.71 & $0.480-$ & 0.634 \\
Inferior perifoveal & 260.95 & 16.56 & 263.55 & 12.39 & $0.0562-$ & 0.577 \\
Nasal perifoveal & 269.90 & 13.12 & 277.60 & 11.65 & $1.962-$ & 0.057 \\
Temporal perifoveal & 245.35 & 15.72 & 247.55 & 12.76 & $0.486-$ & 0.630 \\
\hline
\end{tabular}

CFT showed negative correlation with cumulative dose of HCQ $(r=-0.737)$, table 5

Table (5) Correlation between central foveal thickness and Cumulative dose in grams.

\begin{tabular}{lcc}
\hline & \multicolumn{2}{c}{ Cumulative dose (grams } \\
p value
\end{tabular}

Table (6) Correlation between parafoveal thickness and Cumulative dose in HCQ group.

\begin{tabular}{lll}
\hline & \multicolumn{2}{l}{ Cumulative dose (grams } \\
& $\mathbf{R}$ & $\mathbf{p}$ value \\
\hline Superior parafoveal & -0.645 & 0.001 \\
Inferior parafoveal & -0.691 & 0.001 \\
Nasal parafoveal & -0.746 & 0.001 \\
Temporal parafoveal & -0.668 & 0.001 \\
\hline
\end{tabular}


Table (7) Correlation between CFT and parafoveal thickness with treatment duration in HCQ group.

\begin{tabular}{lcc}
\hline & \multicolumn{2}{c}{ Treatment duration } \\
& $\mathbf{R}$ & P value \\
\hline central foveal thickness & -0.631 & 0.003 \\
superior parafoveal & -0.516 & 0.020 \\
inferior parafoveal & -0.379 & 0.099 \\
nasal parafoveal & -0.324 & 0.163 \\
temporal parafoveal & -0.547 & 0.013 \\
\hline
\end{tabular}

\section{Discussion}

Hydroxychloroquine (HCQ) is the antimalarial product extensively used in rheumatic illnesses such as rheumatoid arthritis and systemic lupus erythematosus. It is most often used for retinopathy and careful screening after SD-OCT [6].

The precise mechanism of retinal toxicity is unclear; in our research, OCT scans revealed a very significant photoreceptor $(\mathrm{p}<0.001)$ affinity for outer retinal components in the foveal and parafoveal regions.

We agree with Chen that external retinal structures including photoreceptors and RPE cells have been affected [4].

While Bulut research suggests that retinal toxicity is related to retinal ganglion (GCL) cell destruction, internal plexiform layer (IPL), or RNFL [7].

Currently, not all patients with SD-OCT anomalies have experienced visual impairments. Interestingly, the ONL thickness was lowered in all $3 \mathrm{D}$ scans even in the patient with the mildest SD-OCT alterations. Those alterations could be seen in regions with normal ophthalmoscopy, Fundus Autoflurescence, and mfERG reactions were also normal. Future assessment of the thickness of ONL during HCQ therapy may thus give even early suspicion of retinal degeneration. [3].

The Kellner research concurred with us that photoreceptive layers changes have been observed in individuals with HCQ retinopathy detected by SDOCT in the form of photoreceptor IS losses evidenced by decreased ONL thickness and disruption, or absence of the IS/OS photoreceptor junction. These structural changes of the retinal complement the observed photoreceptor malfunction with mfERG, a rise in the concentration of lipofuscine and a reduction in the RPE melanin content [8].

Statistical differences with respect to foveola (CFT $1 \mathrm{~mm}$ ) and parafoveal (foveola $3 \mathrm{~mm}$ ) thickness were clearly observed in average $199.50 \mu \mathrm{m} \pm 29.22$ thickness and CFT P value 0.001. For parafoveal quadrants, $\mathrm{P}$ for superior quadrant was 0.010 with average thickness of $289.75 \mu \mathrm{m} \pm 15.81$, p with a mean thickness of 0.004 and $280.35 \mu \mathrm{m} \pm 18.59$, p with average thicknesses of $269.20 \pm 20.60$, and 0.001 with an average thickness of $\mathrm{p}$ for the temporal quadrant, $276.25 \pm 30.44$.

Jonathan research has agreed with us that SDOCT can measure retinal thickness in the event of early retinal HCQ poisoning. And that, if measured against a certain landmark, the retinal thinning and structural anomalies occurring in early poisoning may be more clearly identified: $1.0 \mathrm{~mm}$ from foveola. It also seems that retinal thinning precedes the loss of both IS/OS and RPE dysfunction [9].

In addition, the parafoveal thinning produced by HCQ was consistent with what Korah and Thomas had discovered in our research. They observed that parafoveal retinal thickness measures and volume measurements may proves retinal toxicity, and OCT measurements can be helpful for the early identification of HCQ maculopathy as part of HCQ retinal toxicity screening [10]

In our research, three patients acquired just central foveal dilution, five patients exhibited mixed central foveal and parafoveal dilution, in which the 4 parafoveal quadrants were nearly equally impacted by time quadrate with the most common disease. Although the perifoveal quadrants were not extensively impacted, the lower quadrant exhibited most affection. This requires additional study with a bigger population size.

Histological findings have indicated, however, that peri-foveal photoreceptor cells are most impacted and that they may have a secondary effect owing to RPE disturbance [11].

HCQ is a 4-aminoquinolone known to disturb the lysosomal function of RPE by subsequent photorecipient degeneration resulting to an enhanced accumulation of lipofuscin and therefore a change of the RPE metabolism may play a role in retinal toxicity[12].

Sisternes Study concurred with us that reported SDOCT results in the parafoveal area as localised retinal thinning and verified early toxinity by loss of the IS/OS segment line [13].

\section{Conclusion}

We evaluated the examination findings of the spectral domain optical coherence tomography in patients receiving hydroxychloroquine for more than 2 years as opposed to the gender-based control unit. The statistically significant dilution of the central foveal and para-foveal areas was reported in the hydroxychloroquine group as compared with the control group. SD-OCT may thus be utilised by ophthalmologists in patients who employ $3 \mathrm{D}$ macula and 5 lines scanning mode 1 and $3 \mathrm{~mm}$ and $5 \mathrm{~mm}$ of foveola as a screening tool. SD-OCT is an objective technique of evaluation that provides precise measures of retinal thickness and layers in addition to 
its speed, specificity and availability in most eye centres. On the basis of our research findings, we suggest that SD-OCT be used as a screening device before and after patients take HCQ as a therapy for rheumatic illnesses.

\section{References}

[1] M. F. Marmor, "Comparison of screening procedures in hydroxychloroquine toxicity," Arch. Ophthalmol., vol. 130, pp. 461-469, 2012.

[2] F. Wolfe and M. F. Marmor, "Rates and predictors of hydroxychloroquine retinal toxicity in patients with rheumatoid arthritis and systemic lupus erythematosus," Arthritis Care Res. (Hoboken)., vol. 62, pp. 775-784, 2010.

[3] J. A. Rodriguez-Padilla. "High-speed ultrahigh-resolution optical coherence tomography findings in hydroxychloroquine retinopathy," Arch. Ophthalmol., vol. 125, pp. 775-780, 2007.

[4] E. Chen et al., "Spectral domain optical coherence tomography as an effective screening test for hydroxychloroquine retinopathy (the 'flying saucer' sign)," Clin. Ophthalmol. (Auckland, NZ), vol. 4, pp. 1151, 2010.

[5] O. Ugwuegbu et al., "Quantitative assessment of outer retinal layers and ellipsoid zone mapping in hydroxychloroquine retinopathy," Br. J. Ophthalmol., vol. 103, pp. 3-7, 2019.

[6] M. P. Wiącek, D. Bobrowska-Snarska, W. Lubiński, M. Brzosko, and M. Modrzejewska, "What is new in recommendations on ophthalmological screening in patients treated with chloroquine and hydroxychloroquine? Update and literature review," Niger. J. Clin. Pract., vol. 20, pp. 919-923, 2017.

[7] M. Bulut, E. Muhammet Kazım, D. Toslak, M. Akidan, E. K. Başar, and H. F. Cay, "A new objective parameter in hydroxychloroquineinduced retinal toxicity screening test: macular retinal ganglion cell-inner plexiform layer thickness," Arch. Rheumatol., vol. 33,pp. 52, 2018.

[8] U. Kellner, A. B. Renner, and H. Tillack, "Fundus autofluorescence and mfERG for early detection of retinal alterations in patients using chloroquine/hydroxychloroquine," Invest. Ophthalmol. Vis. Sci., vol. 47, pp. 3531-3538, 2006.

[9] J. B. Kahn, I. D. Haberman, and S. Reddy, "Spectral-domain optical coherence tomography as a screening technique for chloroquine and hydroxychloroquine retinal toxicity," Ophthalmic Surgery, Lasers Imaging Retin., vol. 42, pp. 493-497, 2011.

[10] S. Korah and T. Kuriakose, "Optical coherence tomography in a patient with chloroquineinduced maculopathy," Indian J. Ophthalmol., vol. 56, pp. 511, 2008.

[11] K. E. Stepien, D. P. Han, J. Schell, P. Godara, J. Rha, and J. Carroll, "Spectral-domain optical coherence tomography and adaptive optics may detect hydroxychloroquine retinal toxicity before symptomatic vision loss," Trans. Am Ophthalmol. Soc., vol. 107, pp. 28, 2009.

[12] S. P. Sundelin and A. Terman, "Different effects of chloroquine and hydroxychloroquine on lysosomal function in cultured retinal pigment epithelial cells," Apmis, vol. 110, pp. 481-489, 2002.

[13]L. de Sisternes, J. Hu, D. L. Rubin, and M. F. Marmor, "Localization of damage in progressive hydroxychloroquine retinopathy on and off the drug: inner versus outer retina, parafovea versus peripheral fovea," Invest. Ophthalmol. Vis. Sci., vol. 56, pp. 3415-3426, 2015 . 